\title{
The unmasking of a Brugada phenotype after pulmonary resection: A case report and implications for postoperative management
}

\author{
Saila P. Nicotera, MD, MPH, Min P. Kim, MD, and Malcolm M. Decamp, MD, Boston, Massachusetts
}

The unmasking of latent arrhythmogenic diseases after general thoracic surgery has, to date, not been described. We present a patient in whom a Brugada pattern became evident on electrocardiogram after pulmonary resection.

\section{CLINICAL SUMMARY}

A 78-year-old white man was diagnosed with non-small cell lung cancer by transthoracic fine-needle aspirate. His medical history included type I diabetes mellitus, hypothyroidism, emphysema, and a distant history of cholangiocarcinoma treated with surgery, chemotherapy, and radiation. He had a 30-pack-year smoking history and stopped using tobacco 20 years ago.

Staging evaluation, including magnetic resonance imaging of the brain, positron emission tomography-computed tomography scan, flexible bronchoscopy, and mediastinoscopy, revealed node-negative poorly differentiated stage IA non-small cell lung cancer. The patient enrolled in a clinical trial studying the efficacy of pre-resection administration of amiodarone in reducing postoperative atrial dysrhythmias. As part of the study protocol 7 days before his operation, the patient commenced taking $200 \mathrm{mg}$ of amiodarone orally twice per day. He underwent an uncomplicated left thoracotomy and left lower lobectomy, and continued to receive oral amiodarone the day after his operation. Pain control was achieved using epidural anesthesia, with the catheter positioned at the T4 level. The epidural solution consisted of $0.1 \%$ bupivacaine and $10 \mathrm{mg} / \mathrm{mL}$ of hydromorphone, infused from 4 to $6 \mathrm{~mL} / \mathrm{h}$.

The patient's postoperative course was complicated by oliguria, azotemia, hyperglycemia, and hyperkalemia. Blood urea nitrogen and serum creatinine levels increased to 39 and $1.8 \mathrm{mg} / \mathrm{dL}$ from baseline values of 22 and $0.9 \mathrm{mg} / \mathrm{dL}$, respectively. Peak serum potassium level was measured at 6.1 $\mathrm{mg} / \mathrm{dL}$. On discovery of hyperkalemia, an electrocardiogram was obtained to assess for electrolyte-related changes. "Cove' -type ST elevations and a right bundle branch block were noted in precordial leads V1 and V2. Known as the Brugada syndrome pattern, these findings were vastly different

\footnotetext{
From the Beth Israel Deaconess Medical Center, Harvard Medical School, Boston, Mass.

Received for publication July 3, 2007; accepted for publication Oct 19, 2007

Address for reprints: Saila Pillai Nicotera, MD, MPH, Beth Israel Deaconess Medical Center, Harvard Medical School, Boston, MA 02215 (E-mail: snicoter@bidmc. harvard.edu).

J Thorac Cardiovasc Surg 2008;136:1593-5

$0022-5223 / \$ 34.00$

Copyright (c) 2008 by The American Association for Thoracic Surgery

doi:10.1016/j.jtcvs.2007.10.089
}

from the patient's preoperative electrocardiogram (Figures 1 and 2).

A medication review was undertaken. Any medications known to block cardiac sodium channels were identified and discontinued. The hyperkalemia was corrected. Serial cardiac enzymes failed to reveal an acute ischemic event. A transthoracic echocardiogram showed a mildly dilated left atrium, normal regional left ventricular wall motion, a left ventricular ejection fraction of more than $55 \%$, and trivial mitral regurgitation. Compared with the patient's preoperative study, the echocardiogram was unchanged. Serial electrocardiograms were obtained, and 48 hours after the Brugada pattern was noted, the electrocardiogram normalized.

Throughout the period of electrocardiographic abnormality, our patient remained asymptomatic. He denied any history of syncope or near-syncope. His family history was negative for sudden cardiac death. He recovered from surgery and was discharged on the sixth postoperative day.

\section{DISCUSSION}

Brugada syndrome is a rare inherited arrhythmogenic disease linked to sudden cardiac death, known to be caused most often by mutation of the SCN5A gene located on chromosome $3 \mathrm{p}$. The gene locus codes for a sodium channel protein, and the loss-of-function mutation results in decreased sodium conduction across the channel. Drugs causing sodium channel blockade further reduce channel conduction, precipitating an often fatal dysrhythmia through a reentrant phenomenon.

Several factors have been reported to unmask a latent Brugada electrocardiogram pattern, including fever, electrolyte disturbances, and various pharmacologic agents. ${ }^{1}$ A number of these factors were relevant to our patient. He was receiving bupivacaine via epidural anesthesia. There is 1 case report in the literature in which bupivacaine was postulated to have unmasked a Brugada pattern. $^{2}$

There is 1 recent report in the English language literature of amiodarone inducing a Brugada pattern during the acute intravenous phase of infusion. ${ }^{3}$ Ours is the first known report of the association of oral amiodarone with unmasking the Brugada pattern. Amiodarone slows cardiac conduction via blockade of sodium channels and may be the mechanism by which the phenotype is exposed. Hyperkalemia has been cited as potentially inducing Brugada syndrome, and our patient did have elevated serum potassium levels. ${ }^{4}$

Supraventricular dysrhythmias are a common complication after both cardiac and general thoracic surgery. Our case report is the first known description in the English 
Age: 77 Room: F205

Male White $\star \star *$ CONSIDER ACUTE ST ELEVATION MI $\star * \star *$

Sinus rhythm.

Anteroseptal ST elevation, in leads V1-V2 CONSIDER ACUTE ANTEROSEPTAL INEARCT

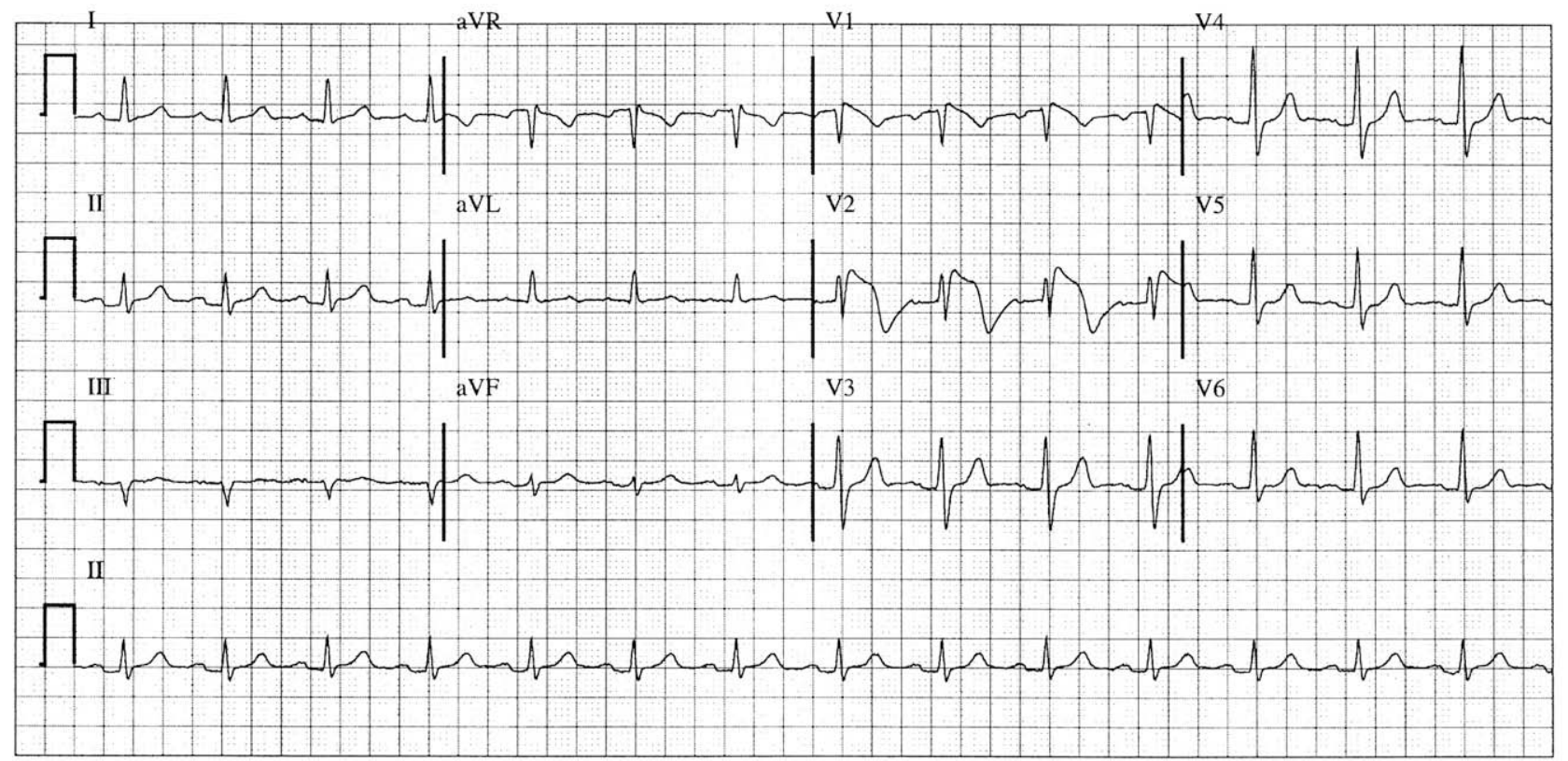

Beth Israel Deaconess Medical Center

$$
\begin{array}{rrr}
\multicolumn{3}{r}{\text { Axes }} \\
\text { P } & \text { QRS } & \text { T } \\
30 & 1 & 4
\end{array}
$$


Age: 77 Room: F205

Sinus rhythm

Normal ECG

Male White

Compared to previous tracing, anteroseptal ST segment elevation has resolved

$1: 07: 12 \quad 08 / 27 / 2006$

Cart 58-37 TR-1110750

Rate Intervals Axes

$\begin{array}{crrrrrr}\text { Rate } & \text { PR } & \text { QRS } & \text { QT/QTC } & \text { P } & \text { QRS } & \text { T } \\ 75 & 154 & 106 & 392 / 418 & 39 & -1 & 6\end{array}$

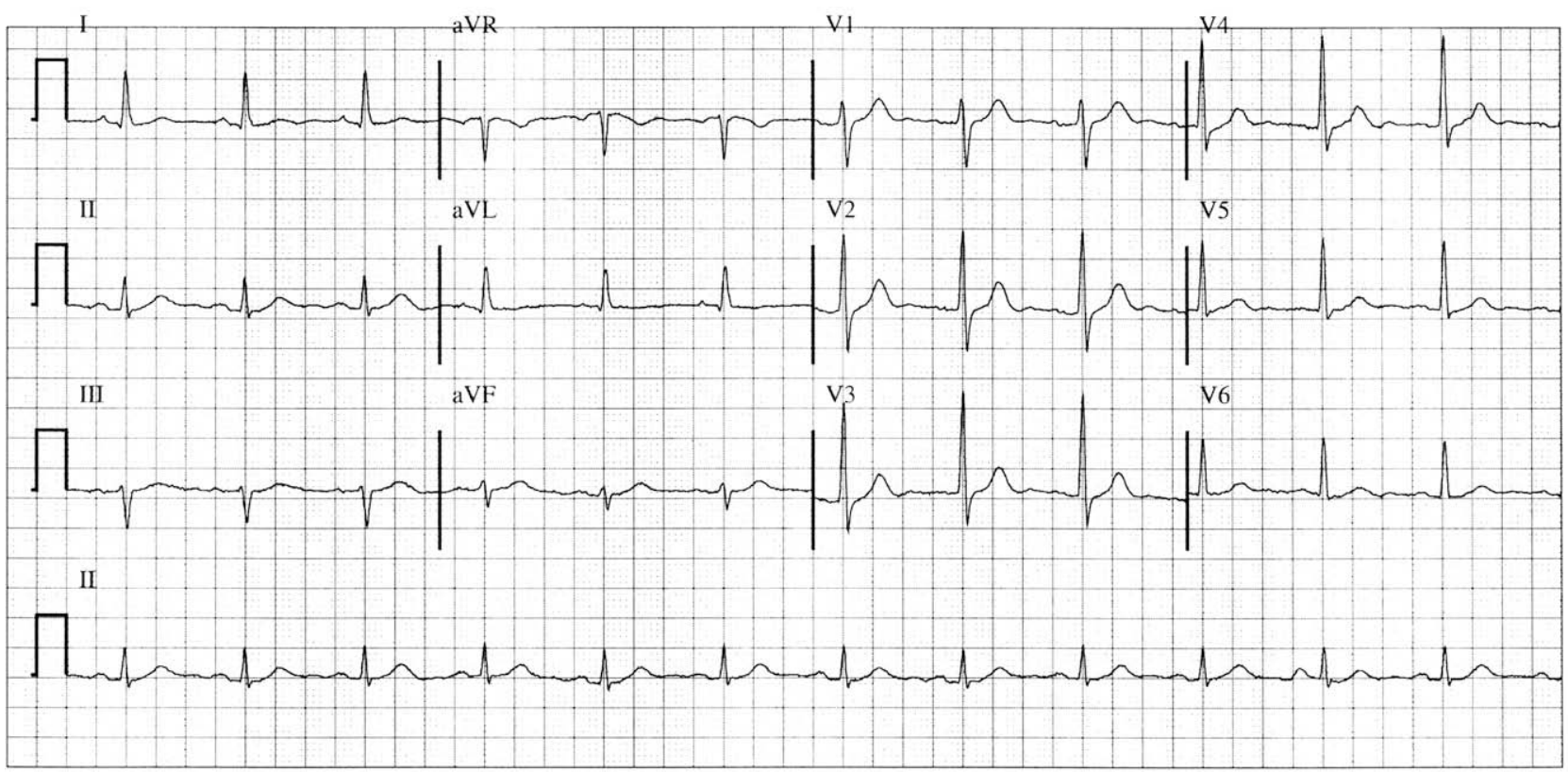

Beth Israel Deaconess Medical Center

$25 \mathrm{~mm} / \mathrm{sec}, 10 \mathrm{~mm} / \mathrm{mV}$

FIGURE 2. Normal rhythm subsequently restored. 